\title{
Variations saisonnières des populations de mirides du cacaoyer dans la région de l'Indénié-Djuablin en Côte d'Ivoire
}

\author{
Norbert N'Dri KOUAME ${ }^{12 *}$, François Kouamé N'GUESSAN ${ }^{1}$, Hauverset Assiénin N'GUESSAN ${ }^{12}$, \\ Pierre Walet N'GUESSAN 1 , Yao TANO 2 \\ ${ }^{1}$ Laboratoire d'Entomologie, Centre National de Recherche Agronomique (CNRA), 01 BP 808 Divo 01, Côte \\ d'Ivoire ; \\ 2. Laboratoire de Zoologie et Biologie Animale, UFR Biosciences, Université Félix Houphouët Boigny de Cocody, 22 \\ BP 582 Abidjan 22, Côte d'Ivoire \\ Auteur pour la correspondance Email : ndri_norbert@yahoo.fr
}

Original submitted in on $4^{\text {th }}$ September 2014. Published online at www.m.elewa.org on 30th November 2014. http://dx.doi.org/10.4314/jab.v83i1.2

\section{RÉSUMÉ}

Objectifs : Les dégâts causés par les mirides dans les cacaoyères constituent l'une des préoccupations majeures des producteurs en Côte d'Ivoire. La présente étude a été réalisée pour déterminer les périodes de fortes pullulations de ces insectes nuisibles dans la région de l'Indénié-Djuablin, une importante zone de production cacaoyère en Côte d'Ivoire

Méthodologie et résultats : L'étude a été menée dans les cacaoyères des producteurs de la région de l'Indénié-Djuablin en 2009-2013. Les variations des populations des mirides ont été évaluées par échantillonnage 2 fois par mois à l'aide de la technique de bâchage. Cette technique a consisté à étaler des bâches de $16 \mathrm{~m}^{2}$ de superficie à l'aplomb de 12 cacaoyers repérés dans une zone infestée par les mirides. Les cacaoyers ainsi repérés et bâchés sont traités avec un insecticide homologué (Califan super $40 \mathrm{EC}$ ) à forte dose et les mirides sont dénombrés sur les bâches 5 heures après le traitement, à chaque passage d'échantillonnage. Les données de pluviométrie et de température ont été collectées également deux fois par jour (matin et soir) à la station de Recherche du Centre National de Recherche Agronomique à Abengourou. Les résultats ont révélé la présence de 4 espèces de mirides dans la région. II s'agit de Sahlbergella singularis, Distantiella theobromae et Bryocoropsis laticollis, communément appelé punaises ou poux du cacaoyer, et Helopeltis spp. désigné sous le nom de moustique du cacaoyer. Sahlbergella singularis s'est avérée l'espèce prédominante $(95,6 \%)$. L'analyse de la courbe de pullulation des mirides de 2009 à 2013 a révélé une seule période de pullulation dans la région qui s'étend de Juillet à février chaque année, avec un pic de population en septembre ou en août selon les années. Par ailleurs, on note que la pullulation des mirides commence pendant les périodes pluvieuses et s'arrête progressivement avec les périodes sèches. A l'inverse, la relation entre les variations des populations des mirides et la température n'était pas évidente. Cependant, les périodes de forte pullulation semblent coïncider avec les températures les plus basses de l'année, la population décroit progressivement à partir de février et mars lorsque la température est plus élevée.

Conclusion et application: Cette étude a permis de connaitre les différentes espèces de mirides en présence et les périodes de fortes pullulations dans la région de l'Indénié-Djuablin. Ces résultats 


\section{Kouame et al. J. Appl. Biosci. 2014 Variations saisonnières des populations de mirides du cacaoyer}

dans la région de l'Indénié-Djuablin en Côte d'Ivoire

permettront de faire des recommandations quant aux périodes favorables aux interventions phytosanitaires. En effet, une application rationnelle d'insecticide doit être effectuée pendant les périodes où les mirides sont actifs dans les plantations.

Mot clés : Cacaoyer, mirides, dynamique des populations, Sahlbergella singularis.

Seasonal variations in the cocoa mirid bugs populations in the region of Indénié-Djuablin in Ivory Coast

\section{ABSTRACT}

Objectives: Damage caused by the mirid bug in cocoa plantations is one of the major concerns of Ivorian cocoa producers. This study was conducted to identify periods of heavy outbreaks of the cocoa mirid bug in the Indenié-Djuabiln region, an important cocoa producing area in Côte d'Ivoire.

Methodology and Results: The study was conducted in farms in the Indénié - Djuablin region in 2009-2013. Seasonal variations of cocoa mirid population were evaluated by sampling twice a month using the knockdown technique. This technique consisted in spreading sheets of $16 \mathrm{~m}^{2}$ in size underneath 12 cocoa trees infested by the mirid bug. The selected trees were treated with a high dose of a registered insecticide (Califan super $40 \mathrm{EC}$ ) and dead mirid bugs were counted on the sheets 5 hours after treatment. Rainfall and temperature data were also collected twice daily (morning and afternoon) at the station of National Centre for Agricultural Research in Abengourou. The results revealed the presence of four mirid species in the region. These species were Sahlbergella singularis, Distantiella theobromae and Bryocoropsis laticollis commonly refrred to as capsids or cocoa mirids, and Helopeltis spp. Known as the cocoa mosquito. Sahlbergella singularis was the predominant species (95.6\%). The analysis of the mirid outbreak curve from 2009 to 2013 revealed only one outbreak period in the region. This period extends from July to February each year, with a peak population in September or August depending on the year. Moreover, the mirid outbreak starts during the rainy season and progressively stops with the onset of the dry season. Although a clear relation was not found between mirid population variation and temperature, the lowest temperatures recorded in the year coincide with the outbreak period and mirid population start decreasing from February and March when the temperature increases.

Conclusion and application: This study allowed knowing the different mirid species in the Indénié-Djuablin region, as well as the outbreak periods. These results will enable to make recommendations with regard to timing of phytosanitary measures. Indeed, a rational application of insecticide should be performed during periods where mirid number increases in the cocoa farms.

Keyword : Cocoa, mirid, population dynamic, Sahlbergella singularis.

\section{INTRODUCTION}

Introduit en Côte d'Ivoire par les colons Européens vers 1900 (Boni, 1985), le cacao joue aujourd'hui un rôle socio-économique primordial en Côte d'Ivoire. Les grandes zones de productions du cacao ivoirien sont l'Est, le Sud-Est, le Centre, le Centre-Ouest, l'Ouest et le Sud-Ouest. La région de l'Indénié-Djuablin située à l'Est produit en moyenne 102284 tonnes de cacao chaque année. Elle constitue la troisième région de production de Cacao en Côte d'Ivoire (Assiri, 2010). La culture du cacaoyer est compromise par plusieurs contraintes biotiques. II s'agit de maladies, d'attaques d'insectes nuisibles, et de dégâts de rongeurs et de plantes parasites (Kébé et al., 2006). Au regard des insectes nuisibles, les attaques de mirides constituent la préoccupation majeure des producteurs (Lavabre, 1961; Coulibaly et al, 1998).Plus de 1000 genres et 10 000 espèces de mirides sont connus dans le monde (Babin, 2009), toutefois, environ 40 espèces s'attaquent au cacaoyer. Ces espèces sont phytophages et appartiennent toutes à la sous-famille des Bryocorinae. En Côte d'Ivoire, 4 genres appartenant à deux tribus ont été identifiés 


\section{Kouame et al. J. Appl. Biosci. 2014 Variations saisonnières des populations de mirides du cacaoyer}

dans la région de l'Indénié-Djuablin en Côte d'Ivoire

dans la cacaoyère. Ce sont Sahlbergella, Distantiella et Bryocoropsis de la tribu des Odoniellini et Helopeltis de la tribu des Monaloniini (N'Guessan et Coulibaly, 2000). Les dégâts primaires causés par ces insectes résultent de l'action de la piqûre sur les rameaux et les fruits
(Figure 1). La salive injectée intoxique et détruit les cellules végétales. Sur les pousses tendres, les piqûres de mirides sont suffisantes pour tuer l'organe. Dans ces conditions, les feuilles brunissent et demeurent attachés au rameaux.
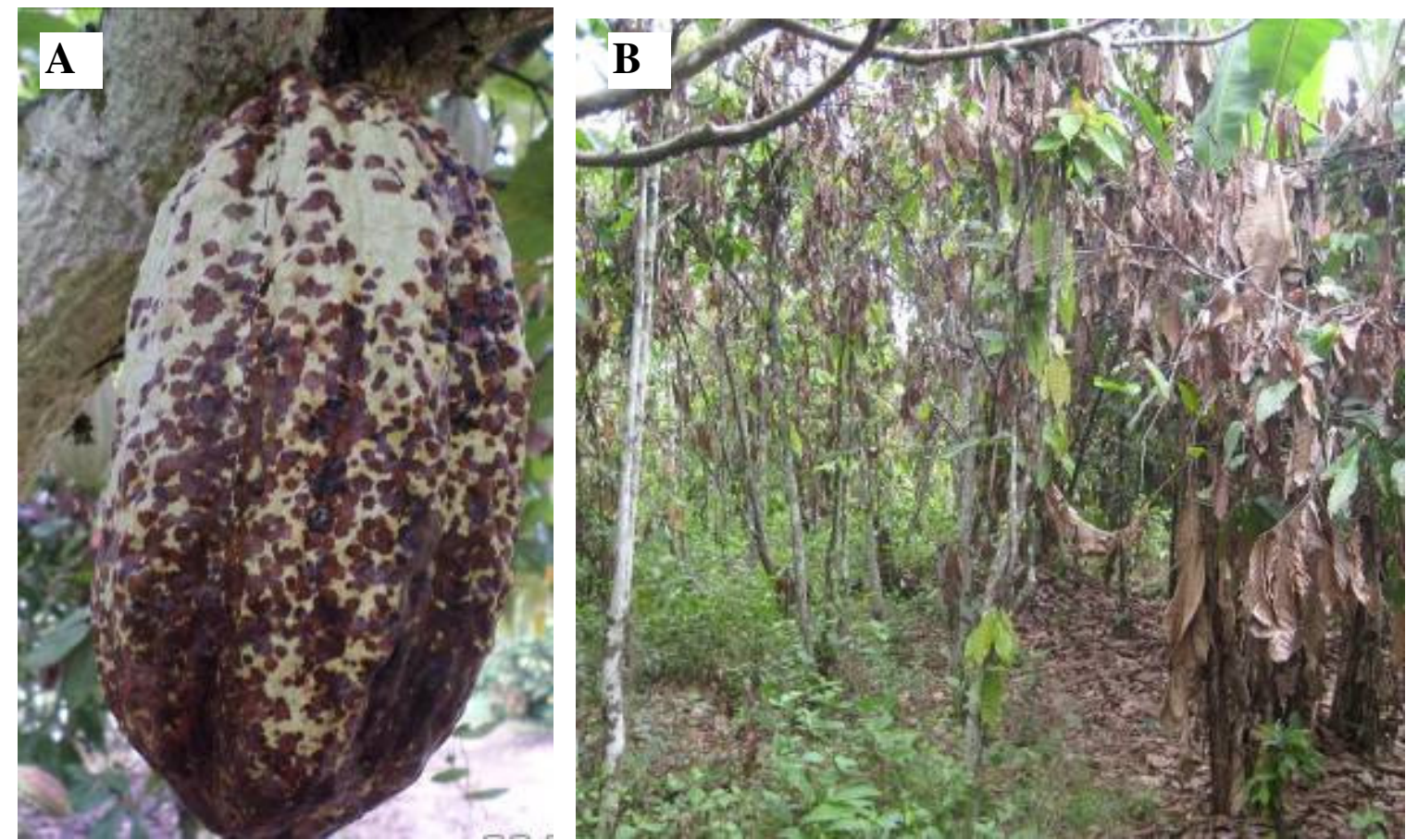

Figure 1 : Dégâts de mirides (A - sur cabosse ; B - Sur rameaux).

Quand aux jeunes cabosses, elles se nécrosent et tombent. Toutefois, la plus grande part des pertes de production dues à ces mirides provient des attaques sur les rameaux et les branchettes (Lavabre, 1992; Babin, 2009). En effet, les dégâts secondaires sont dus à un champignon appelés Calonectria rigidiuscula. Ce champignon intervient sur les tissus blessés ou nécrosés. Dans les cas d'attaques chroniques, la frondaison du cacaoyer s'amenuise d'année en année et les extrémités des branches se dessèchent (Lavabre, 1992). Les dégâts causés par les mirides conduisent à une dégradation partielle ou totale de la cacaoyère et à une réduction du potentiel de production des arbres. Les pertes annuelles dues aux attaques de mirides peuvent atteindre 30 à $40 \%$ du rendement (Entwistle, 1972; Lavabre, 1977a). Le principal moyen de lutte contre ces déprédateurs demeure
I'utilisation d'insecticides (Kebe et al., 2005; N'Guessan, 2005). En Côte d'lvoire, les périodes de traitements sont basées sur les variations saisonnières des populations de mirides. Les premiers travaux sur la biologie, l'écologie des mirides et la dynamique des populations des mirides ont été réalisés dans les années 60 (Lavabre, 1961). En raison des perturbations climatiques de nos jours et des inefficacités des traitements insecticides observés en milieu paysan, il était nécessaire d'actualiser les informations sur les variations saisonnières des populations de ces insectes dans les principales régions de production. C'est dans ce contexte que la présente étude a été menée dans la région de I'Indénié-Djuablin, avec l'objectif de déterminer les périodes de fortes pullulations des mirides. 


\section{Kouame et al. J. Appl. Biosci. 2014 Variations saisonnières des populations de mirides du cacaoyer}

dans la région de l'Indénié-Djuablin en Côte d'Ivoire

\section{MATERIELS ET METHODE}

Site d'étude : L'étude a été réalisée dans la région de l'Indénié-Djuablin, située dans la zone Est de la Côte $d^{\prime}$ Ivoire, entre les latitudes $5^{\circ} 45$ et $7^{\circ} 10$ Nord et les longitudes $3^{\circ} 10$ et $3^{\circ} 50$ Ouest. La région est couverte de forêts denses mésophiles dans sa partie nord et de forêts denses ombrophiles dans sa partie sud. Le climat est de type tropical humide et est caractérisé par 4 saisons de durée inégale (Brou, 2005; Kouamé et al., 2006). II s'agit d'une grande saison des pluies de mimars à mi-juillet, d'une petite saison pluvieuse qui se situe entre septembre et novembre, d'une grande saison sèche qui débute en décembre et prend fin en mi-mars et enfin, d'une petite saison sèche peu marquée qui se rencontre dans le mois d'août. La précipitation moyenne annuelle est de 1241,45 mm. La région de l'Indénié-Djuablin constitue l'ancienne boucle du cacao et fournit aujourd'hui en moyenne $8 \%$ de la production nationale chaque année.

Méthode d'échantillonnage: Trois localités représentatives de la zone de production de cacao de la région de l'Indénié-Djuablin ont été choisies (Aniassué, Abengourou et Niablé) pour l'échantillonnage des mirides. Dans chaque localité, 2 sites constitués par des blocs de plusieurs cacaoyères voisines attaquées par les mirides ont été identifiés, soit au total six blocs de cacaoyères. Ces blocs de cacaoyères avaient pour sites centres les plantations de Monsieur Diarra Tiemoko et Madame Namké Ahouman Juliette pour la localité d'Abengourou, deux différentes plantations de Monsieur Sansan Kouaho pour la localité de Niablé, et les plantations de Messieurs Issouf Illa et Koffi Kouassi Bertin pour la localité d'Aniassué. Ces sites centres, avec les cacaoyères voisines situées dans le même périmètre avec un rayon de 2 à $3 \mathrm{~km}$ représentaient les sites d'échantillonnage. Sur chaque site d'échantillonnage, le niveau d'infestation des cacaoyères par les mirides a été évalué 2 fois par mois dans la première et la troisième semaine de chaque mois par la technique de bâchage. A cet effet, des bâches de $16 \mathrm{~m}^{2}$ de

\section{RESULTATS}

Espèces de mirides collectées: L'échantillonnage réalisé dans la région de l'Indénié-Djuablin a permis de collecter 4 espèces de mirides. Ce sont Sahlbergella singularis $\mathrm{Hgl}$ (1895), Distantiella theobromae Dist (1909), Bryocoropsis laticollis Schum (1917) et Helopeltis sp. (Figure 2). L'analyse de variance a révélé des différences significatives $(P<0.05)$ entre les différentes espèces de mirides au regard des niveaux superficie sont étalées à l'aplomb de 12 cacaoyers repérés dans une zone infestée par les mirides. Ainsi, à chaque passage d'échantillonnage, 24 cacaoyers sont bâchés par localités, soit 24 échantillons. Les cacaoyers repérés et bâchés sont traités avec un insecticide homologué. L'insecticide utilisé est le Callifan super $40 \mathrm{EC}$ à la concentration de $250 \mathrm{ml}$ de produit commercial pour 12 litres d'eau pour tuer tous les insectes hébergés par les cacaoyers bâchés. A chaque passage d'échantillonnage, les traitements ont été effectués tôt les matins entre $7 \mathrm{~h}$ et $8 \mathrm{~h}$. Le produit est appliqué sur la base de 12 litres de bouillie insecticide pour 12 arbres. Les arbres déjà traités sont marqués avec de la peinture rouge et les traitements effectués à des dates ultérieures se faisaient en dehors des endroits touchés par les traitements antérieurs.

Observations: Un dénombrement de mirides morts sur les bâches a lieu 5 heures après le traitement insecticide. A cet effet, les cacaoyers bâchés étaient secoués pour faire tomber tous les insectes morts retenus dans le feuillage. Les mirides ont été ramassés à l'aide de pinces entomologiques souples, placés dans des boîtes de Pétri et ensuite dénombrés en distinguant les différentes espèces à l'aide de la clé de détermination établie par Lavabre $(1970 ; 1977 a)$. Le nombre de mirides a été déterminé par arbre et par mois pour chaque site d'échantillonnage. Les données de précipitations et de température ont été collectées au cours de l'étude pour tenir compte des variations des conditions climatiques au cours de l'étude.

Analyse des données: Les données collectées ont été soumises à une analyse de variance à l'aide de la procédure GLM ('General Linear Model') de SAS (Anonyme, 2009) en vue de comparer les populations des différentes espèces de mirides en présence. La séparation des moyennes a été réalisée par le test de Student-Newman-keuls au seuil de $5 \%$. La moyenne mensuelle des populations des mirides a été également calculée et représentée graphiquement en vue de déterminer les périodes de fortes pullulations.

de population (Tableau 1). L'espèce Sahlbergella singularis $\mathrm{Hgl}$ s'est avérée l'espèce prédominante $(95,6 \%)$ dans la région. Les espèces Bryocoropsis laticollis Schum, Distantiella theobromae Dist et Helopeltis sp sont présentes, mais faiblement représentées, soit respectivement $1,25 \%, 2,06 \%$ et $1,1 \%$. 

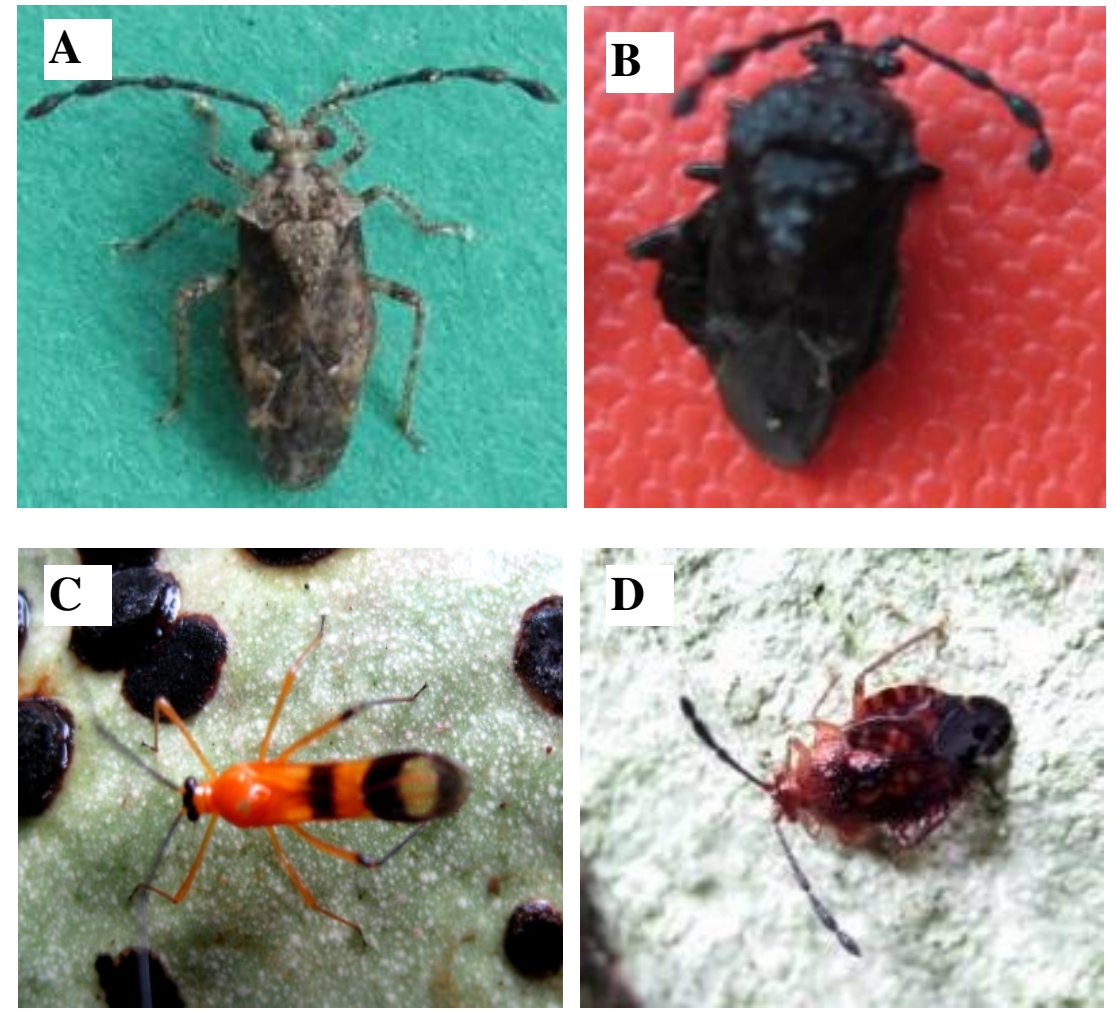

Figure 2: Espèces de mirides collectées dans la région de l'Indénié-Djuablin (A- Sahlbergella singularis Hgl, BDistantiella theobromae Dist, C- Helopeltis sp, D- Bryocoropsis laticollis Schum).

Tableau 1: Population moyenne de 4 espèces de mirides dans la région de l'Indénié-Djuablin

\begin{tabular}{lc}
\hline Espèce de miride & Population moyenne \\
\hline Sahlbergella singularis & $82,58 \pm 11,70 \mathrm{a}$ \\
Distantiella theobromae & $1,78 \pm 0,39 \mathrm{~b}$ \\
Bryocoropsis laticollis & $1,08 \pm 0,21 \mathrm{~b}$ \\
Helopeltis sp & $0,95 \pm 0,21 \mathrm{~b}$ \\
\hline
\end{tabular}

Les moyennes suivies d'une même lettre sont statistiquement identiques au seuil de 5\% (Student-Newman-keuls)

L'analyse des courbes de pullulations des mirides des localités d'Abengourou, Niablé et Aniansué (Figure 3) permet de distinguer une seule période de forte pullulation. Cette période s'étend de juillet à février pour les trois localités. La période de juillet 2010 à janvier 2011 montre une importante activité des mirides dans la localité d'Abengourou avec un pic de population qui 65 mirides par arbre. Cependant, les données des 4 années d'observation montrent révèlent une plus forte pullulation des mirides dans la localité de niablé par rapport aux autres localités (Tableau 2). La courbe moyenne de pullulation des mirides (Figure 4) confirme cette seule période de fortes pullulations de juillet à février dans la région de l'Indénié-Djuablin. Cette courbe révèle un niveau de population des mirides élevé dans les mois d'Août, Septembre, Octobre, Novembre et Décembre. Les pics de population sont généralement enregistrés dans les mois d'août et septembre chaque année avec des niveaux de population atteignant 18 à 30 mirides par arbre. Dans les mois de Février, Mars, Avril, Mai et Juin, la population est relativement faible avec des niveaux de 2 à 4 mirides par arbre (Figure 4). Les résultats cumulés des quatre années d'observation montrent une importante activité des mirides du cacaoyer pendant la période de Juillet à Décembre avec un pic en Septembre (Figure 5). 
Kouame et al. J. Appl. Biosci. 2014 Variations saisonnières des populations de mirides du cacaoyer dans la région de l'Indénié-Djuablin en Côte d'Ivoire

Tableau 2. Population moyenne des mirides dans 3 localités de la région de l'Indénié-Djuablin

\begin{tabular}{lc}
\hline Localité & Mirides moyens par arbre \\
\hline Niable & $10,13 \pm 1,30 \mathrm{a}$ \\
Abengourou & $5,67 \pm 1,31 \mathrm{~b}$ \\
Anuansué & $5,43 \pm 0,87 \mathrm{~b}$ \\
\hline Les moyennes suivies d'une même lettre sont statistiquement identiques au seuil de $5 \%$ (Student-Newman-keuls).
\end{tabular}

L'analyse de l'évolution de la hauteur des pluies au cours des 4 années d'étude révèle une seule période pluvieuse au cours de l'année. Cette période s'étend de février à novembre (Figures 4 et 5). Cependant, en 2011, 2012 et 2013 une baisse de la hauteur de pluie a été notée dans les mois de juillet et août. L'analyse de la courbe de pullulation des mirides en relation avec le régime pluviométrique montre que la pullulation commence pendant les périodes pluvieuses et s'arrête progressivement avec les périodes sèches. Les températures moyennes des quatre années d'observation ont varié de $22,3^{\circ} \mathrm{C}$ à $28^{\circ} \mathrm{C}$. Les faibles températures se situent au niveau des mois de juillet, août et septembre. Les températures les plus élevées se situent en février et mars. A l'inverse de la pluviométrie, la relation entre les variations des populations des mirides et la température n'était pas évidente. Cependant, les périodes de forte pullulation semblent coïncider avec les températures les plus basses de l'année. En effet, la population décroit progressivement à partir de février et mars lorsque la température est plus élevée (Figure 6).

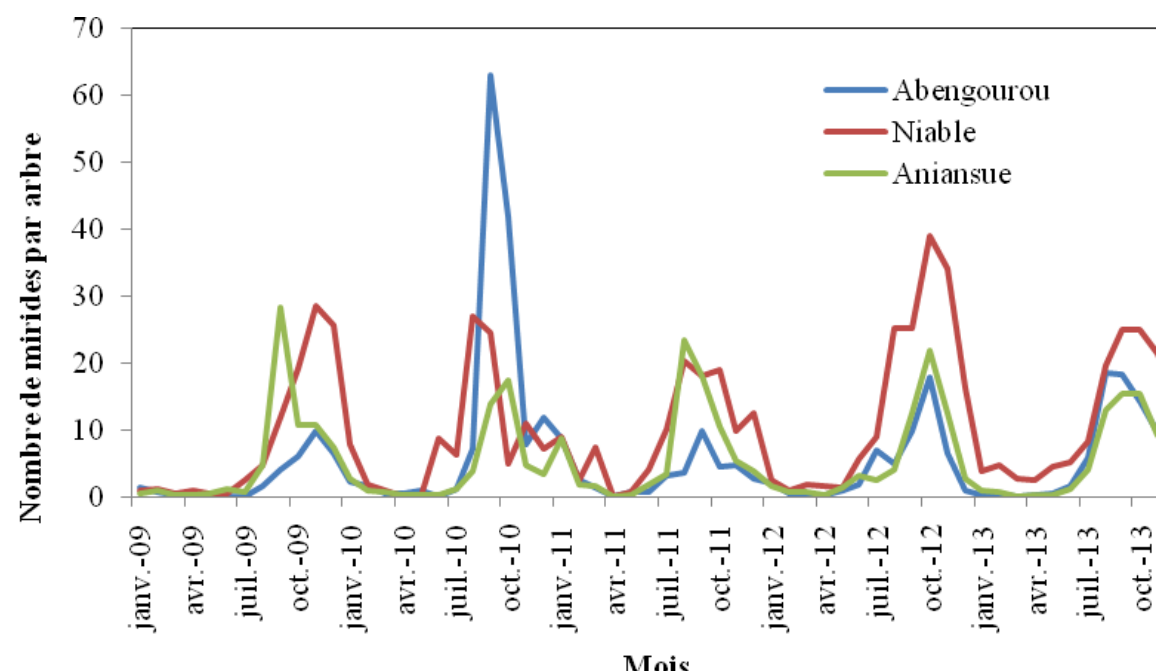

Figure 3: variation saisonnière de la population de mirides dans trois localités de la région de l'Indénié-Djuablin en Côte d'Ivoire. 
Kouame et al. J. Appl. Biosci. 2014 Variations saisonnières des populations de mirides du cacaoyer dans la région de l'Indénié-Djuablin en Côte d'Ivoire

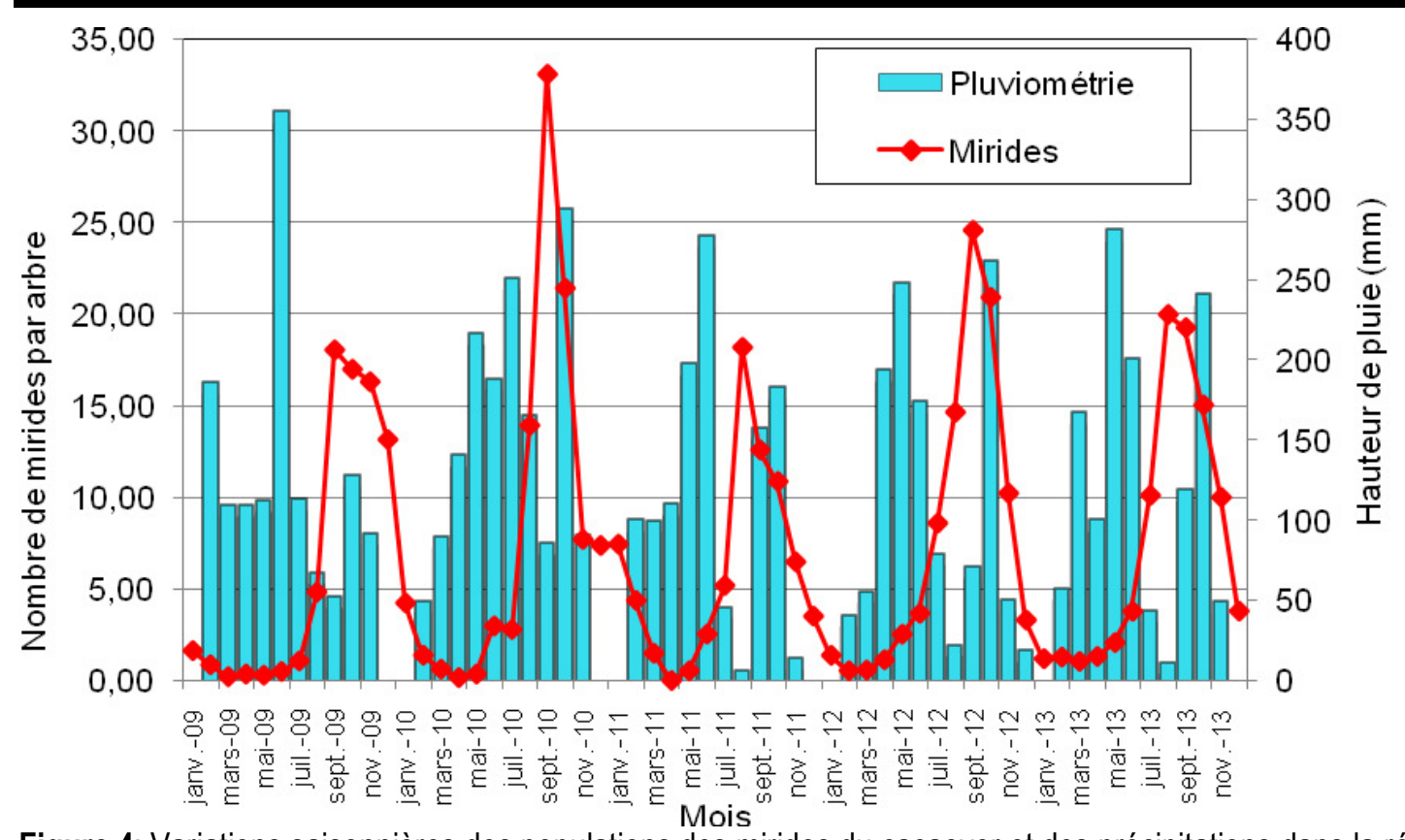

Figure 4: Variations saisonnières des populations des mirides du cacaoyer et des précipitations dans la région de l'Indénié-Djuablin en Côte d'Ivoire.

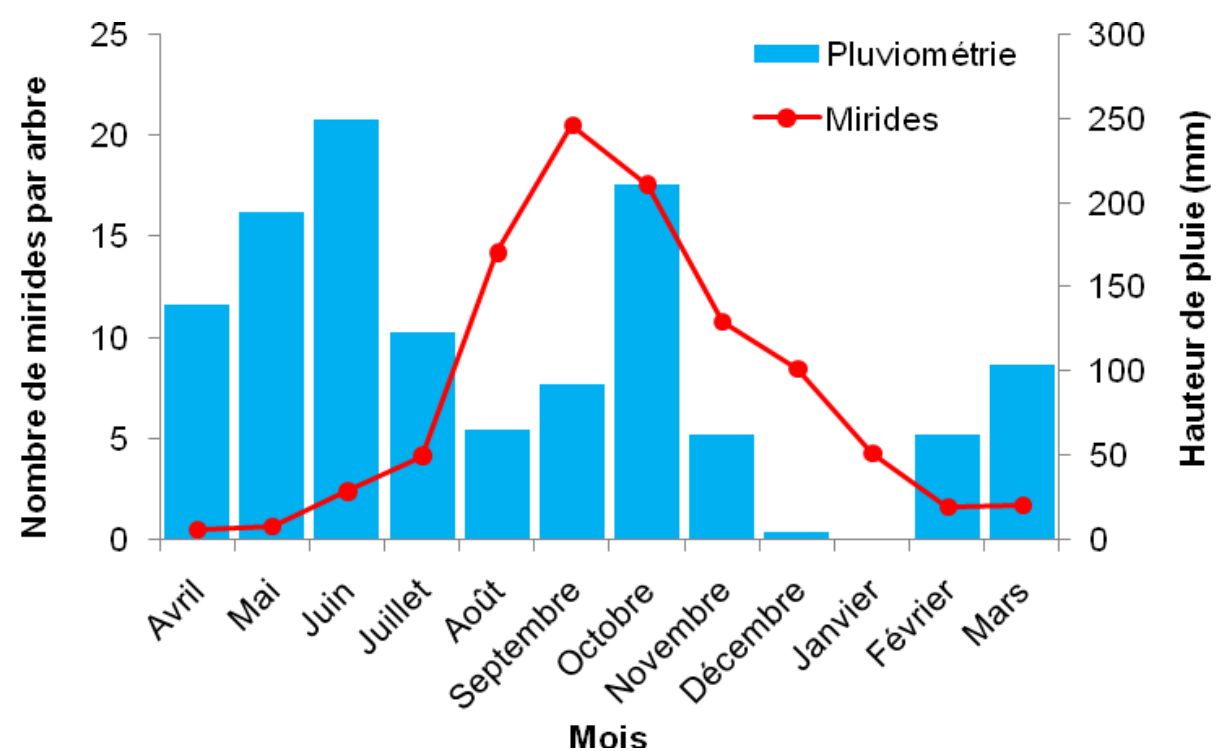

Figure 5: Variations mensuelle des populations des mirides du cacaoyer et des précipitations dans la région de l'Indénié-Djuablin en Côte d'Ivoire. 


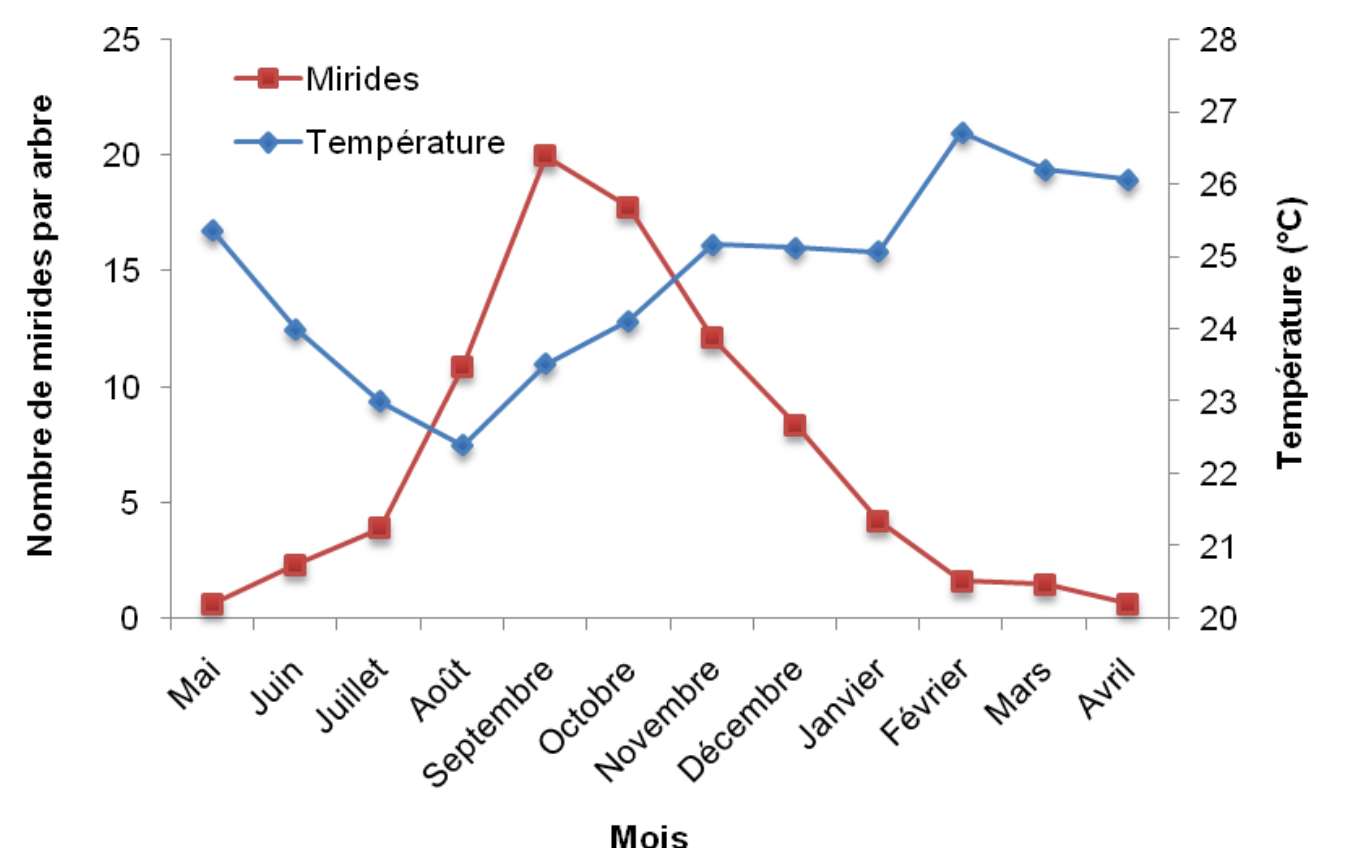

Figure 6 : Variations mensuelle des populations des mirides du cacaoyer et de la température dans la région de l'Indénié-Djuablin en Côte d'Ivoire.

\section{DISCUSSION}

La connaissance des périodes de pullulation des mirides est une partie intégrante de la lutte contre ces déprédateurs. Le suivi des variations saisonnières des populations de ces insectes dans la région de l'IndéniéDjuablin a révélé la présence des 4 espèces de mirides connues dans le verger de cacaoyers en Côte d'Ivoire (N'Guessan et Coulibaly, 2000). L'espèce Sahlbergella singularis $\mathrm{Hgl}$ (1895) était l'espèce la plus répandue dans la région avec un niveau de population très élevé que celui des autres, soit $95,6 \%$ des mirides collectés dans la région. Ces résultats corroborent ceux de Nguyen-Ban (1977) et N'Guessan (2005) qui ont indiqué que Sahlbergella singularis est l'espèce prédominante dans le verger cacaoyer de la Côte d'Ivoire. La prédominance de Sahlbergella singularis par rapport aux autres espèces a été également notée dans les autres pays producteurs, notamment au Ghana et au Cameroun (Lavabre, 1977b). Au regard des variations mensuelles et annuelles des populations des mirides, les résultats de Cette étude ont révélé une seule période de forte pullulation dans la région de l'Indenié-Djuablin. Cette période s'étend de juillet à février chaque année. Ces résultats sont contraires à ceux de Lavabre (1977a) qui avaient affirmé que la règle générale de fluctuation des populations est l'existence de deux périodes bien tranchées. Ces deux périodes seraient liées, l'une à la grande saison des pluies et l'autre à la petite saison. En effet, dans les années 60s, Lavabre et al. (1963) avaient indiqué que dans les régions d'Abengourou et de Divo où on notait deux saisons pluvieuses bien distinctes, il existait deux pics de pullulation de mirides consécutifs aux périodes pluvieuses. Dans Cette étude une seule période de pluie a été enregistrée au cours de l'année, ce qui pourrait expliquer la présence dune seule période de pullulation. En effet, Brou (2005) et Kouamé et al. (2006) ont indiqué que ces dernières années, la petite saison sèche est peu marquée dans la région de l'Indénié-Djuablin. L'existence d'une seule période pluvieuse peut être donc liée aux perturbations climatiques de ces dernières années. En effet, le climat qui était de type bimodal en Côte d'Ivoire dans la plupart des zones de production sauf à l'ouest a certainement subit des modifications en raison des perturbations climatiques (Morel, 2004). Plusieurs études ont mis en évidence les impacts directs ou indirects des changements climatiques sur les insectes nuisibles (Gagnon, 2011 ; Plouffe et al., 2012 ). Outre les variations saisonnières enregistrées, il semble exister des différences dans l'intensité des pullulations des mirides selon les années et les localités. En effet, le pic de population des mirides étaient de 18 mirides par arbre en septembre 2009, 33 en septembre 2010, 18 en août 2011, 25 en septembre 2012 et 20 mirides 


\section{Kouame et al. J. Appl. Biosci. 2014 Variations saisonnières des populations de mirides du cacaoyer}

dans la région de l'Indénié-Djuablin en Côte d'Ivoire

par arbre en août 2013. Ce phénomène de variation du niveau de population des mirides d'une année à l'autre avait déjà été signalé par Lavabre et al. (1963) dans le Centre-Ouest à Koréa et Zéprégué et dans le CentreSud à Heremankono, où il y avait une différence nette entre les populations de mirides de 1961 et celles de 1962. Cela peut s'expliquer par des variations dans les facteurs qui influent sur les fluctuations cycliques des populations de mirides (Lavabre et al., 1963; Bruneau De Miré, 1970; Decazy, 1974). L'effet de la pluviométrie et de la température sur les variations saisonnières des populations de mirides ont fait l'objet de recherche par Lavabre et al. (1963), Bruneau De Miré (1970) et Decazy (1974). Ces auteurs avaient démontré que les pluies ne pouvaient pas être considérées comme un facteur agissant sur les variations de populations des mirides ; et que par ailleurs, il n'existait pas de relation de causalité entre les fluctuations de température et celles des populations de mirides. Cependant, Lavabre et al. (1963) ont indiqué que l'accroissement du nombre de mirides est plutôt sous la dépendance d'une revitalisation végétative qui favorise un renouveau qualitatif et quantitatif de la sève du cacaoyer. Ainsi, la principale cause du phénomène de fluctuation des populations de mirides est constituée par les facteurs trophiques, notamment les fruits et les gourmands. Fohrer (2011) a indiqué que chez les insectes en général, l'apport de nourriture peut favoriser l'implantation, la prolifération et la dissémination. Cependant, il faut noter que chez le cacaoyer, les facteurs trophiques eux-mêmes sont conditionnés par les facteurs climatiques, notamment la pluviométrie qui favorise les repousses et la production des fruits. De même, les fortes températures peuvent être à l'origine de déshydratation des tissus et d'une réduction de la quantité de sève qui est la principale nourriture des mirides. Cette étude a révélé que le niveau des populations de mirides de la région de l'IndéniéDjuablin est très faible de Janvier à Juin et élevé de Juillet à Décembre. Cette situation pourrait s'expliquer par la présence ou l'absence de nourriture pour ces déprédateurs selon les mois. Par ailleurs, dans les mois de juillet et août, on note une baisse sensible de la pluviométrie et un début de progradation des populations des mirides. Cet accroissement du nombre

\section{CONCLUSION}

D'une manière générale, il ressort de Cette étude que l'espèce de mirides la plus dominante de la région de l'Indénié-Djuablin est Sahlbergella singularis Hgl (1895) et que la période de juillet à février constitue les de mirides peut s'expliquer par un enrichissement en eau des plantes dû à la forte pluviosité de février à juin. En effet, le nombre de fruits qui constituent la principale source d'alimentation des mirides est faible de Janvier à Juin et élevé de Juillet à Décembre (Decazy, 1977). Néanmoins, selon Entwistle (1972), chez S. singularis et $D$. theobromae qui n'ont pas de préférence particulière pour les cabosses, l'accroissement de la population n'est pas étroitement lié à la présence des fruits sur les arbres. Cette espèce se nourrit et se développe aussi bien sur les gourmands et les branchettes. Outre les facteurs climatiques qui agissent directement ou indirectement sur les populations des insectes, l'action des ennemis naturels peut être à l'origine des variations cycliques des populations des déprédateurs. Quoique, l'action des ennemis naturels sur la dynamique des populations des mirides n'ait pas été prise en compte dans Cette étude, Entwistle (1972) et Collingwood (1977) avaient identifié plusieurs parasitoïdes, prédateurs et champignons entomopathogènes susceptibles de réduire les populations de ces insectes dans les cacaoyères. Entwistle (1972) a indiqué que les larves d'un hyménoptère (Leiophron sahlbergellae Wlk) se développent dans les larves de stades 2 à 5 de Sahbergella singularis et contribue ainsi à réguler sa population. De même plusieurs espèces de fourmis, notamment Oecophylla longinoda (Latr.), Tetramorium aculeatus (Mayr), Camponotus spp, Crematogaster spp, Polyrachis spp, Pheidole spp et Platythyrea spp. ont été inventoriés comme des prédateurs des mirides. Par ailleurs, Collingwood (1977) avait identifié quelques espèces de champignons entomopathogènes (Hirsutella sp. et Beauveria sp.) qui s'attaquaient à des populations naturelles de S. singularis. Récemment, Padi et al. (2001) ont isolé des souches des Beauveria bassiana à partir de $S$. singularis au Ghana. Tous ces ennemis naturels peuvent contribuer aux variations mensuelles et annuelles des densités des mirides dans les cacaoyères. Toutefois, des observations précises doivent être effectuées sur ces agents potentiels de lutte biologique pour élucider leur rôle dans la régulation des populations des mirides dans les cacaoyères.

moments d'intenses activités de ces insectes. Ces résultats devraient permettre de faire des recommandations quant aux périodes favorables d'applications éventuelles d'insecticides. Nos résultats 


\section{Kouame et al. J. Appl. Biosci. 2014 Variations saisonnières des populations de mirides du cacaoyer}

dans la région de l'Indénié-Djuablin en Côte d'Ivoire

ont permis d'actualiser les connaissances sur la dynamique des populations dans la région de l'IndéniéDjuablin, et constituent des données de bases nécessaires à la mise en œuvre d'un programme de lutte contre les mirides dans cette région. En effet, les populations de mirides peuvent éventuellement être maintenues à un niveau bas en saison humide lorsque

\section{REMERCIMENTS}

Les auteurs remercient Messieurs Dosso Loua, Kouadio Kouassi Armand et Gnahoua Olivier Pacôme, techniciens du laboratoire d'entomologie de la Station de Recherche CNRA de Divo, pour leur participation à la réalisation des travaux. Les auteurs expriment

\section{REFERENCES}

Assiri A. A., 2010. Étude d'itinéraires techniques pour la réhabilitation et la replantation cacaoyères dans 10 départements des régions Est, SudEst et Centre-Ouest de la Côte d'lvoire. Doctorat de l'Université de Cocody, 163p.

Anonyme., 2009. SAS/STAT User's guide, Version 9.2, SAS Institute Inc.

Babin R., 2009. Contribution à l'amélioration de la lutte contre le miride du cacaoyer Sahlbergella singularis Hagl. (Hemiptera: miridae). Influence des facteurs agro-écologiques sur la dynamique des populations du ravageur. Doctorat de l'Université Paul Valéry, Montpellier III, 246 p.

Boni D., 1985. Aspects géographiques du binôme café cacao dans l'économie ivoirienne. Les Nouvelles Editions Africaines, $111 \mathrm{p}$.

Brou Y T., 2005. Climat, mutations socio-économiques et paysages en Côte d'lvoire. Mémoire de synthèse des activités scientifiques présenté en vue de l'obtention de l'habilitation à Diriger des Recherches. Université des Sciences et Technologies de Lille, France, 212p.

Bruneau de mire P., 1970. Observations sur les fluctuations saisonnières d'une population de Sahlbergella singularis au Cameroun. Café Cacao Thé. 14, 202-207.

Collingwood C. A., 1977. Biological control and relations with other insects. In : Les Mirides du cacaoyer. Institut français du café et du cacao: 237- 255.

Coulibaly N., N'Guessan F. K., Decazy B., Medus D., Aidara S., Coulibaly A., 1998. Le Fumivap : une nouvelle technique d'application des produits chimiques dans la lutte contre les les traitements insecticides ont lieu pendant les périodes où les populations sont réduites à leur minimum. De même, une application rationnelle d'insecticide peut être effectuée pendant les périodes de fortes pullulations où les mirides sont actifs dans les plantations.

également leur gratitude au FIRCA qui a financé ces travaux pour le compte de la filière café cacao en Côte d'Ivoire. Cet article est publié avec la permission du Directeur Général du Centre National de Recherche Agronomique.

mirides du cacaoyer en côte d'ivoire. Agronomie Africaine, 10 (1): 23-31.

Decazy B., 1974. Les variations saisonnières des populations de Boxiopsis madagascariensis Lavabre, miride ravageur du cacaoyer à Madagascar. Notepréliminaire. Café Cacao Thé 18: 255-262.

Decazy B., 1977. Les mirides du cacaoyer à Madagascar: Boxiopsis madagascariensis Lavabre. In: Les Mirides du cacaoyer. Institut Français du Café et du Cacao: 123 - 137.

Entwistle P F., 1972. Pests of cocoa. Longman Group Ltd, London, United Kingdom; 779 P.

Fohrer F., 2011. Le diagnostic des infestations en milieu patrimonial: approches techniques et méthodologiques. La lettre de l'OCIM numéro 138, novembre-décembre, $40 \mathrm{p}$.

Gagnon A E., 2011. Étude de cas pour évaluer l'impact des changements climatiques en phytoprotection. Colloque en agroclimatologie. $4 \mathrm{p}$.

Kebe I. B., Koffi N., Konan A., N'Guessan K. F., Kohi N. J., Irie B. Z., 2005. Bien cultiver le cacaoyer en Côte d'Ivoire. CNRA, 4 p.

Kebe I. B., Koffie K., N'Guessan K. F., Assiri A. A., Adiko A., Ake S. \& Anno P A., 2006. Le Swollen Shoot en Côte d'lvoire: situation actuelle et perspectives. In: $15^{\text {ème }}$ conférence internationale sur la recherche cacaoyère, San Jose (Costa Rica), volume II: 907-922

Kouame B., Kone D., Yoro G R., 2006. La pluviométrie en 2005 et 2006 dans la moitié Sud de la Côte d'Ivoire. Bulletin le CNRA en 2006, document technique: 12-13. 
Lavabre E. M., 1961. Protection des cultures de caféier, cacaoyer et autres plantes pérennes tropicales. Institut français du café et cacao, Paris, $268 \mathrm{p}$.

Lavabre E M., Decelle J., Deborb P., 1963. Étude de l'évolution régionale et saisonnière des populations de Mirides (Capsides) en Côte d'Ivoire. Caf' Cacao Thé 7: 267-287.

Lavabre E. M., 1970. Insectes nuisibles des cultures tropicales. G.P Maisonneuve et Larose, $276 \mathrm{p}$.

Lavabre E. M., 1977a. Importance économique des mirides dans la cacaoculture mondiale. In: Les Mirides du cacaoyer. Institut Français du Café et du Cacao: $141-153$.

Lavabre E. M., 1977b. Variations saisonnières des populations. In: Les Mirides du cacaoyer. Institut Français du Café et du Cacao:155-170.

Lavabre E. M., 1992. Ravageurs des cultures tropicales. Le Technicien d'Agriculture Tropicale; Maisonneuve \& Larose. 178 p.

Morel R., 2004. Le climat et l'implantation des hommes: le cas de la Côte d'Ivoire. Annales de l'Association Internationale de Climatologie, Vol 1: 117-132.

N'Guessan K. F., 2005. Lutte contre les mirides et les autres insectes nuisibles du cacaoyer en Côte d'lvoire. In: formation des agents du FDPCC sur les techniques de culture, de protection et de traitements post-récoltes du cacao en Côte d'Ivoire. CNRA, cahier du stagiaire: 28-48.

N'Guessan K. F. \& Coulibaly N., 2000. Dynamique des populations de mirides et de quelques autres déprédateurs du cacaoyer dans la région Ouest de la Côte d'lvoire. Actes de la 13ème conférence internationale sur la recherche cacaoyère. Kota Kinabalu, Sabah (Malaisie): 425-429.

Nguyen-ban J., 1977. La lutte chimique contre les mirides du cacaoyer. In Les Mirides du cacaoyer. Institut français du café et du cacao: 257-278.

Padi B., Ackonor J. B., Opoku I. Y., 2001. Cocoa IPM research and implementation in Ghana. In: Proceedings of the West Africa Regional Cocoa IPM Workshop, Janny Vos and Peter Neuenschwander Eds. Cotonou, Benin, CPL press, pp 54-62.

Plouffe D., Gaetan B., 2012. Modèles bioclimatiques pour la prévision des risques associés aux ennemis des cultures dans un contexte de climat variable et en évolution. Centre de Référence en Agriculture et Agroalimentaire du Québec. Commission Agrométéorologie (CRAAQ). 13 p. 